\title{
EXPERIMENTING WITH THE MULTIPLE WORLDS CONCEPT TO SUPPORT THE DESIGN OF AUTOMATED CONTAINER TERMINALS
}

\author{
Michele Fumarola \\ Gwendolyn Kolfschoten \\ Alexander Verbraeck \\ Delft University of Technology \\ Jaffalaan 5 \\ 2628BX Delft, THE NETHERLANDS
}

\author{
Cornelis Versteegt
}

APM Terminals Maasvlakte II B.V. Boompjes 40

3011XB Rotterdam, THE NETHERLANDS

\begin{abstract}
The complexity of systems requires design approaches that are more iterative and interactive to explore different design perspectives and to remain flexible for dynamic requirements and design contexts. This paper describes the multiple worlds approach to support multi actor participative design. The environment enables a group to explore, analyze and compare design alternatives based on a simulation of the performance of key components, and the system behavior in a 3D visualization. This environment allows stakeholders to visualize different perspectives as a structured overview of design choices. This helps the stakeholders to create shared understanding and increases the transparency of their decision process. In this paper we present the approach and the results of an experiment to evaluate the way in which the environment supports design.
\end{abstract}

\section{INTRODUCTION}

Xia and Lee (2005) note an increased complexity in a design process in a multi-actor environment due to the number of actors involved in the process, the number of stakes and perspectives present, the different ways the actors relate to each other, the dynamics in the multi-actor environment due to the interaction between actors, and, finally, the uncertainty with respect to actor behavior. During the design process, actors interact with each other and with the systems surrounding them (Van der Lei, Kolfschoten et al. 2010). Pruyt (2010) pinpoints the interaction between actors can lead to complex decision-making processes and to unforeseen/unintended effects. Moreover, different forms of organization of actor interactions and different degrees and forms of participation may lead to different outcomes. One of the most critical challenges of design in a multi actor setting is the creation of shared understanding among actors to increase the quality of design decisions (Piirianen, Kolfschoten et al. 2010).

In engineering, designing systems is part of the field of 'systems engineering' (SE). Either implicitly or explicitly, theories and methods from SE are used in large logistics projects such as container (Agerschou 2004) and airport (Horonjeff and McKelvey 2010) terminals. Over the last couple of decades, SE brought about a change in how technical systems are designed and implemented. SE is a holistic approach, focusing on the whole system instead of concerning on the sole individual parts. By creating a high level overview of a complete engineering process, we can often identify a set of steps such as requirements analysis, design, implementation, and testing. These steps can be taken sequentially with or without feedback possibilities, and one can also iterate through all steps multiple times. Such processes lie at the core of SE and are studied and described by renowned scholars in the field (Sage and Armstrong 2000; Hitchins 2008).

Responding to the trend of increased complexity in system design, SE methods and techniques have become more iterative, interactive and agile, to make the designers flexible with respect to changing requirements (Fumarola, Seck et al. 2010; Fumarola, Seck et al. 2011). Design methods are often 
accompanied by IT supporting tools to visualize the implications of design decisions using forecasts. These tools need to support interactive and iterative design in order to enable design teams to efficiently explore the solution space. In our research, in which we followed the design science methodology, we want to make a contribution to the realm of tools and methods that support designers in forecasting to explore the implications of design decisions. An application domain is which this is especially relevant is logistic systems. The design process of modern logistics systems, such as container terminals, involves various actors. Actors are not only the stakeholders within the system (e.g. company) but also external parties that do profit directly from the system, for instance public opinion and authorities (Donaldson and Preston 1995). In our design science study we identified research opportunities during the relevance cycle by carrying out a case study on the design of automated container terminals. During the rigor cycle (Fumarola, Seck et al. 2011), we identified the conceptual constructs to develop a design method, which we coined as the "multiple worlds" design method. We operationalized this method by applying it in the context of our original case study. To operationalize it, we developed a design environment that uses simulation components (Fumarola, Seck et al. 2010) and a visualization client (Fumarola and Versteegt 2011) to achieve shared understanding. In this paper, we want to present our efforts in evaluating the method in order to identify improvements for the multiple world design method that can be implemented in the design cycle.

\section{THE MULTIPLE WORLDS DESIGN METHOD}

In previous work (Fumarola, Seck et al. 2010), we have introduced the multiple worlds design method that supports the exploration of the solution space in the design of systems. Starting from Simon's decision making paradigm (Simon 1996), which is composed of structuring the problem, evaluating alternatives upon criteria and selecting the best alternative, we concluded that modeling and simulation (M\&S) is often seen as a tool to analyze (Zeigler and Praehofer 2000) thus fitting in the evaluation phase of Simon's paradigm. However, we noted that M\&S could be introduced much earlier in the process in the phase of structuring the problem. M\&S would herewith enhance the decision maker's ability of generating alternatives more comprehensively, which would enable exploring and analyzing each design decision during the synthesis.

Designing systems in a multi-actor environment is complex from the system as well as the actor perspective. Classic operations research and systems engineering provide valuable techniques to analyze systems. Due to an increased interest in facilitating the design process from a system and an actor perspective, we also need insights in how actors behave and how we can support them. For this we build on the literature of Group Decision Support Systems. These systems offer a combination of tools to structure and organize information on a decision to support groups in creating shared understanding and to improve the transparency of decision making (Nunamaker, Briggs et al. 1997). Starting from existing literature (Fumarola, Seck et al. 2011), we have presented the constructs that are needed in a multi-actor design method. The method we propose revolves around a tree of design alternatives. The tree is a structure that is composed of nodes and edges. Each node contains an underspecified design: during the design process, a design under investigation can be further specified by deciding upon structure and parameters of the design. This view on design is given by Churchman, who defines the design of a system as 'the design of components and their relationships' (Churchman 1971). In our case, this definition can be operationalized as "the design of a system is the specification of the structure and parameters of that system." A design is said to be underspecified when one or more sub-structures or parameters are not defined. Each underspecified design needs to have a simulation model that can generate performance indicators of that design. This, of course, poses a challenge: an underspecified design needs a simulation model that reflects the uncertainty attained by the elements of the design that have not been defined. This means that from a single design, a multitude of designs can spring: for every structural decision or parameter that has not been set, the complete domain of that structural decision and parameter is used to vary the input and to estimate the output, which gives us the range of the parameter. 
The tree guides actors or designers through the design process. Each node offers a simulation of an alternative design choice, offering support in visualizing and analyzing this alternative. Each simulation can be used to explore the implications of the design choice, and the environment, allows users to compare these alternatives based on several performance. The outputs of the upper nodes specify ranges that would preferably contain the more restricted ranges of the lower nodes. Ultimately, the design process will be about progressively specifying more about the design that restricts the output range to something that satisfice (in Simon's terms (Simon 1996)) the actors involved in the design process.

To operationalize a design method, we have implemented a design environment that visualizes and simulates designs that have been designed in AutoCAD. The design environment allows inputting designs in AutoCAD that can be structure in the design tree. Each node contains a simulation model that can be run and visualized in a virtual 3D environment. The complete architecture of the environment is visualized in Figure 1. Once the design has been defined in AutoCAD, it is sent to the simulation server that carries out the simulation experiment. The simulation models are automatically generated using a component library developed in D-SOL (Jacobs 2005) using the DEVS formalism (Seck and Verbraeck 2009). The output is visualized using a visualization clients that runs $3 \mathrm{D}$ virtual environment developed using OGRE (Torus Knot Software Ltd 2010).

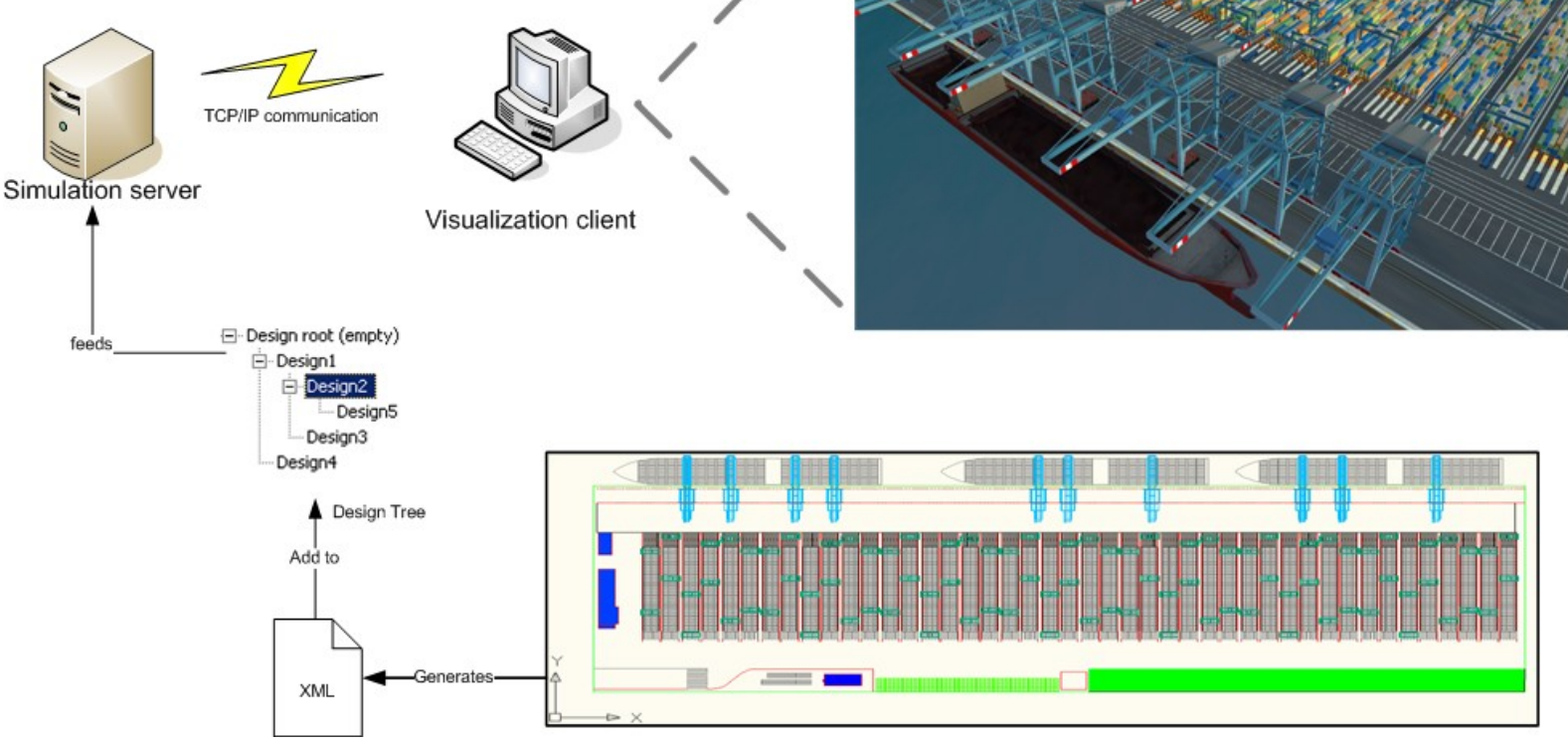

Figure 1: The support environment to design using the Multiple Worlds design method

\section{THE EXPERIMENT}

Following the design science methodology, we describe the design cycle in which we carry out an evaluation to refine the design further. We believe information systems need to be studied in their totality: the supporting information technology and the actors involved in the activity, without diminishing the importance of their personal, social and cultural values. To understand the complete intervention that an information system imposes on an environment, we believe we need to create a rich picture that encompasses the artifact and the actors that are influenced by the artifact, and therefore choose to use a set of qualitative research instruments adhering to an interpretive research tradition.

To carry out the qualitative evaluation, we have organized a workshop with 9 graduate students from Delft University of Technology. The students were divided in groups of 3 to conduct a design study using the proposed method. To simulate a multi-actor environment, we proposed three roles: the business 
analyst, the performance engineer, and the environmental analyst. The roles were accompanied by role descriptions to stimulate the specific behavior expected from that actor. Each role had specific goals that are congruent with reality and each goal was quantified in terms of costs, operational performance indicators or pollution threshold. This subdivision of roles was used to stimulate the discussions and disagreements that are predominant in multi-actor environments.

The experiment was subdivided in 6 phases spread over a 4.5 hours time span. During the first phase, a domain expert from APM Terminals gave a general presentation on the design process of automated container terminals. The presentation thoroughly explained the concepts that are important to understand to design container terminals (such as calculating the required throughput of a terminal given its physical proportions). The various types of equipment, such as cranes and vehicles were presented with technical specifications. During and after the presentation, the participants had the possibility to ask questions to understand the subject matter. During the second phase, a demonstration was given on the support environment and on how to use it. In the third phase the participative design process required the teams to create a high level design specifying the number of equipment and total capital investment of the terminal. The fourth up until the sixth phase consisted of using the detailed simulation model to analyze the performance of the specific functions of the terminal: the loading and unloading on the quay, the horizontal transportation using automated vehicles and the storage at the yard. During the last phase, the participants had the opportunity to freely explore the design space by climbing back up the design tree and try different alternatives for their designs.

Besides the design environment, the participants were equipped with a technical infrastructure to support and stimulate group work. To support the collaboration among multiple participants, we used interactive whiteboards and 3D visualizations. Every group could use 2 interactive whiteboards each connected to a different computer: one for the design environment and on to display an Excel spreadsheet to keep track of the financial, operational, and environmental aspects. The interactive whiteboards were used to display the animation output of the simulation runs and to discuss the designs in AutoCAD. The physical setup and the participants working with the environment are shown in Figure 2 .
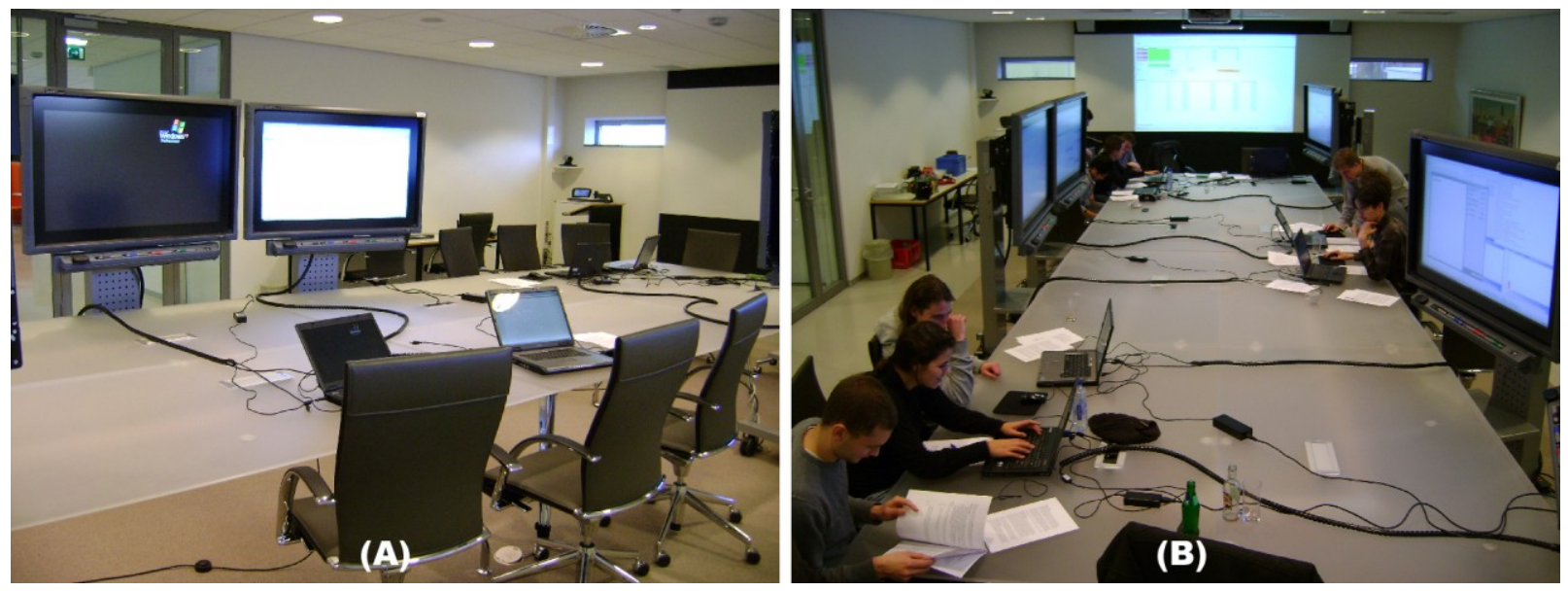

Figure 2: (A) the physical setup for each group of participants, (B) the participants at work

\section{RESULTS}

The main source of data collection for this usability experiment was a semi-structured group interview. To give the participants time to formulate their answers, we prepared a questionnaire using the questions of the semi-structured interview. The following statements were presented and participants could respond on a 7-point Likert scale (from 1 , totally disagree to 7 , totally agree): 
1. The method supported me in inputting from my actor's perspective.

2. The method supported me in protecting both my interests and the collective interests.

3. The method supported me in following the design process without losing sight of my goals.

4. The method supported the construction of enough alternative designs.

5. The method enabled the specification of the decisions using the predefined components and parameters.

6. The method enabled the comparison of designs using the results of the simulation model presented through the animation.

7. The method enabled the comparison of designs using the results of the simulation model presented through the statistics.

Figure 3 shows the results of this questionnaire. From an actor perspective, students indicated that the environment supported the group better than the individual stakeholder. The approach also guided the group in their design effort and helped them to structure their design decisions. Finally, students gave a higher score to the support by statistics than by animation. The semi-structured interview provided more insights on the participant's experiences. There was a consensus on the fact that the method helped cooperate with different actors from different perspectives and that the possibility of exploring different design alternatives helped the participants carrying out their task. However, the software environment to support the method still has some issues. The major problem is AutoCAD: using this design environment, the participants have to follow a strict way of drawing using pre-existing blocks and a certain naming convention. If one deviates from this, the simulation environment cannot run correctly. Another minor issue was the limited output: although the participants could use the statistics and visualization to study the simulation output, they suggested having a more extensive set of statistics to get a more detailed view on the performance of the system.

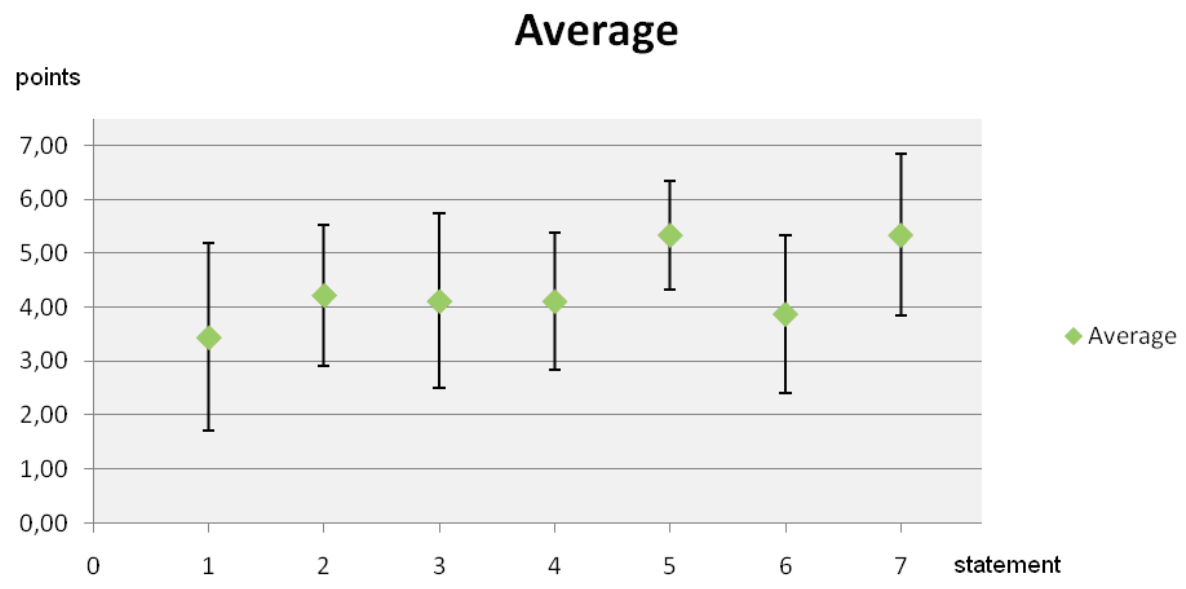

Figure 3: Results of the initial questionnaire on a 7-point Likert scale

\section{DISCUSSION AND CONCLUSION}

In this paper, we have presented our preliminary evaluation of the "multiple worlds" design method. Following the design science methodology, this evaluation is part of the design cycle to identify issues and find possibilities to improve the method.

Initially, we expected that the large number of alternatives would increase the complexity of design study. On the contrary, we found that the participants were effectively using the design tree to structure their decision process and to follow the design process. The participants constructed a large number of design alternatives and used the simulation models to quickly assess the implications of their decisions. This led them to discuss the designs and argue for a certain decision based on a better shared 
understanding of implications of that decision. The animations and statistical results were used to understand the performance of the system from the different actor perspectives, thus helping the group to create a more holistic view of the design choices they faced. Concluding we can state that the multi world method offers a promising environment to enable decision makers to explore design choices from different actor perspectives, to structure their choices and to build shared understanding on the implications of these choices. Further research is required to test this methodology in the field, and to explore opportunities for improvement of the translation of the AUTOCAD drawings.

\section{REFERENCES}

Agerschou, H. 2004. Planning and design of ports and marine terminals. Thomas Telford, Ltd.

Churchman, C. W. 1971. The Design of Inquiring Systems, Basic Concepts of Systems and Organizations. New York, USA, Basic Books.

Donaldson, T. and L. E. Preston. 1995. "The stakeholder theory of the corporation: concepts, evidence, and implications." The academy of Management Review, 20 (1): 65-91.

Fumarola, M., M. Seck, et al. 2010. "A DEVS component library for simulation-based design of automated container terminals." 3rd International ICST Conference on Simulation Tools and Techniques, Torremolinos, Spain, Institute for Computer Sciences, SocialInformatics and Telecommunications Engineering.

Fumarola, M., M. Seck, et al. 2010. "Multiple worlds: a formalism for simulation based design." 2010 Spring Simulation Multiconference. Orlando, USA, ACM.

Fumarola, M., M. Seck, et al. 2011. "Simulation-based systems design in multi-actor environments." Intelligence-based systems engineering. L. Jaim and A. Tolk. Berlin Heidelberg, Springer.

Fumarola, M. and C. Versteegt. 2011. "Supporting automated container terminal design processes with 3D virtual environments." Handbook of Research on Practices and Outcomes in Virtual Worlds and Environment. H. Yang and S. Yuen, eds., IGI Global.

Hitchins, D. K. 2008. Systems engineering: a 21st century systems methodology. New York, NY, USA, John Wiley and Sons, Inc.

Horonjeff, R. and F. McKelvey. 2010. Planning and design of airports, McGraw-Hill Professional.

Jacobs, P. H. M. 2005. "The DSOL simulation suite - Enabling multi-formalism simulation in a distributed context." Delft, Delft University of Technology. Ph.D. Dissertation.

Nunamaker, J. F. J., R. O. Briggs, et al. 1997. "Lessons from a dozen years of group support systems research: a discussion of lab and field findings." Journal of management information systems, 13: 163-207.

Piirianen, K., G. L. Kolfschoten, et al. 2010. In search for the right tools to fix the right problem: a look into the challenge of collaborative design. Hawaii international conference on systems science. Kauai, Hawaii, USA, IEEE Computer society press.

Pruyt, E. 2010. "Multi-actor systems and ethics." International transactions in operational research, 17(4): 507-520.

Sage, A. and J. J. Armstrong. 2000. Introduction to Systems Engineering. New York, NY, USA, John Wiley \& Sons Inc.

Seck, M. and A. Verbraeck. 2009. "DEVS in DSOL: Adding DEVS operational semantics to a generic Event-Scheduling Simulation Environment." SCS 2009 Summer Computer Simulation Conference, The Society for Computer Simulation International.

Simon, H. A. 1996. The Sciences of the Artificial, The MIT Press. 
Torus Knot Software Ltd. 2010. "OGRE - Open Source 3D Graphics Engine." Retrieved April 8th, 2010, from http://www.ogre3d.org.

Van der Lei, T. E., G. Kolfschoten, et al. 2010. "Complexity in multi-actor system research: towards a meta-analysis of recent studies." Journal Design Research, 8 (4): 317-342.

Xia, W. and G. Lee. 2005. "Complexity of information systems development projects: conceptualization and measurement development." Journal of management information systems, 22 (1): 45-83.

Zeigler, B. P. and H. Praehofer. 2000. Theory of modeling and simulation, Academic Press. 